\title{
High-frequency performance of electric field sensors aboard the RESONANCE satellite
}

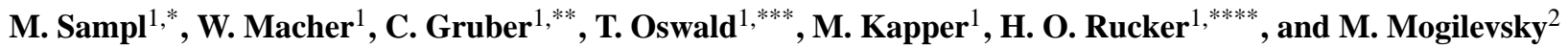 \\ ${ }^{1}$ Space Research Institute, Austrian Academy of Sciences, Graz, Austria \\ ${ }^{2}$ Space Research Institute, Russian Academy of Sciences, Moscow, Russia \\ *now at: KATHREIN-Werke KG, Rosenheim, Germany \\ ** now at: Karl Franzens University Graz, Institute of Physics, Graz, Austria \\ *** now at: Thomas Oswald Aerospace Software, Weinitzen, Austria \\ **** now at: Austrian Academy of Sciences, Commission for Astronomy, Graz, Austria
}

Correspondence to: M. Sampl (manfred.sampl@ieee.org)

Received: 1 August 2014 - Published in Geosci. Instrum. Method. Data Syst. Discuss.: 18 December 2014 Revised: 28 March 2015 - Accepted: 11 April 2015 - Published: 4 May 2015

\begin{abstract}
We present the high-frequency properties of the eight electric field sensors as proposed to be launched on the spacecraft "RESONANCE" in the near future. Due to the close proximity of the conducting spacecraft body, the sensors (antennas) have complex receiving features and need to be well understood for an optimal mission and spacecraft design. An optimal configuration and precise understanding of the sensor and antenna characteristics is also vital for the proper performance of spaceborne scientific instrumentation and the corresponding data analysis. The provided results are particularly interesting with regard to the planned mutual impedance experiment for measuring plasma parameters. Our computational results describe the extreme dependency of the sensor system with regard to wave incident direction and frequency, and provides the full description of the sensor system as a multi-port scatterer. In particular, goniopolarimetry techniques like polarization analysis and direction finding depend crucially on the presented antenna characteristics.
\end{abstract}

\section{The RESONANCE project}

The RESONANCE project is dedicated to the investigation of properties and features of Earth's auroral acceleration zone as well as near-equatorial phenomena. The missions overarching goal is to study wave-particle interactions in the inner magnetosphere. Features to be observed include the energy transfer between energetic particle species, particle precipitation, the magnetospheric cyclotron maser and the generation of planetary radio emissions (Demekhov et al., 2003) such as the auroral kilometric radiation. Comprised of four satellites and flying a magneto-synchronous formation, the mission seems ideal for the investigation of effects and processes which are occurring along the geomagnetic flux tubes. Of particular interest is the energy exchange between the ionospheric and magnetospheric layers of Earth's atmosphere. Compared to previous radio science missions (cf. Table 1; Boudjada et al., 2010), RESONANCE provides the unique feature of sampling the same physical parameters in two space regions belonging to the same magnetic flux tube. The mission features, as well as the proposed spacecraft design, have already been described in Mogilevsky et al. (2002, 2013).

The measurement of electric field parameters in spaceborne radio astronomy poses a substantial challenge, since the observed values range from several $\mathrm{Hz}$ to several $10 \mathrm{~s}$ of $\mathrm{MHz}$ (in case of RESONANCE $10 \mathrm{MHz}$ ) and often comprises a large dynamic range. Gurnett (1998) provided a good overview and showed the large range that instruments usually have to cover in frequency, power, time and spatial scale. This is caused not only by the desire of the mission planers for acquiring a maximized data set, spanning as many phenomena as possible, but also by the fact that the satellite trajectories often pass through regions of different ambient conditions. 
Table 1. Overview of selected science missions and instruments associated with auroral radio emissions

\begin{tabular}{lll}
\hline $\begin{array}{l}\text { Spacecraft } \\
\text { Experiment) }\end{array}$ & $\begin{array}{l}\text { Receiver } \\
\text { features }\end{array}$ & $\begin{array}{l}\text { Stokes } \\
\text { components }\end{array}$ \\
\hline RESONANCE & $\begin{array}{l}0-10 \mathrm{~Hz} \\
10 \mathrm{~Hz}-20 \mathrm{kHz} \\
10 \mathrm{kHz}-10 \mathrm{MHz}\end{array}$ & $\begin{array}{l}\text { I, Q, U, V } \\
\text { Waveform }\end{array}$ \\
\hline $\begin{array}{l}\text { DEMETER } \\
\text { (ICE) }\end{array}$ & DC-3.175MHz & I \\
\hline Cassini & $3.5 \mathrm{kHz}-16.1 \mathrm{MHz}$ & I, Q, U, V \\
(RPWS) & & \\
\hline INTERBALL 2 & $4 \mathrm{kHz}-1 \mathrm{MHz}$ & $\mathrm{I}, \mathrm{Q}, \mathrm{U}, \mathrm{V}$ \\
(POLRAD) & $4 \mathrm{kHz}-500 \mathrm{kHz}$ & \\
\hline Wind & $20 \mathrm{kHz}-1.040 \mathrm{MHz}$ & $\mathrm{I}, \mathrm{V}$ \\
(WAVES) & $1.075 \mathrm{MHz}-13.825 \mathrm{MHz}$ & \\
\hline FAST & $16 \mathrm{~Hz}-2 \mathrm{MHz}$ & $\mathrm{I}$ \\
\hline Voyager (PRA) & $1.2 \mathrm{kHz}-40.2 \mathrm{MHz}$ & $\mathrm{I}, \mathrm{V}$ \\
\hline
\end{tabular}

The scientific instrumentation aboard the RESONANCE spacecraft will include a particle and wave complex, amongst them low- and high-frequency electric field sensors quasi-DC (direct current) to HF (high frequency). The highfrequency analyzer sensors (HFA) ranging from $10 \mathrm{~Hz}$ to $10 \mathrm{MHz}$ and their supporting booms are analyzed in this project. The block diagram shown in Fig. 1 provides an overview of the instrument's layout. HFA consists of cylindrical sensors (so-called $B$-antennas, labeled as ED1-ED4 in Fig. 1) mounted on the tip of four boom rods (so-called $A$ antennas) which extrude from the central body of the spacecraft. Furthermore, the boom rods themselves are used together with the cylindrical sensors for mutual impedance measurements of the ambient plasma environment. The exact configuration is not yet completely fixed due to open questions about the optimum arrangement, including electromagnetic as well as mechanical considerations.

\section{Previous work and methods}

In a related paper, Sampl et al. (2012), we already described the properties of the antenna system in the quasi-static frequency range, which were acquired by rheometry measurements and numerical computations. The herein presented computer simulations provide the characteristics of the antenna system from the quasi-static border up to $40 \mathrm{MHz}$, containing the proposed instrument's operational range (up to about $10 \mathrm{MHz}$ ).

Numerical analysis of the sensor system for frequencies above the quasi-static regime provide the sought characteristics, where experimental techniques such as rheometry (Rucker et al., 1996; Oswald et al., 2009) or in-flight calibra-

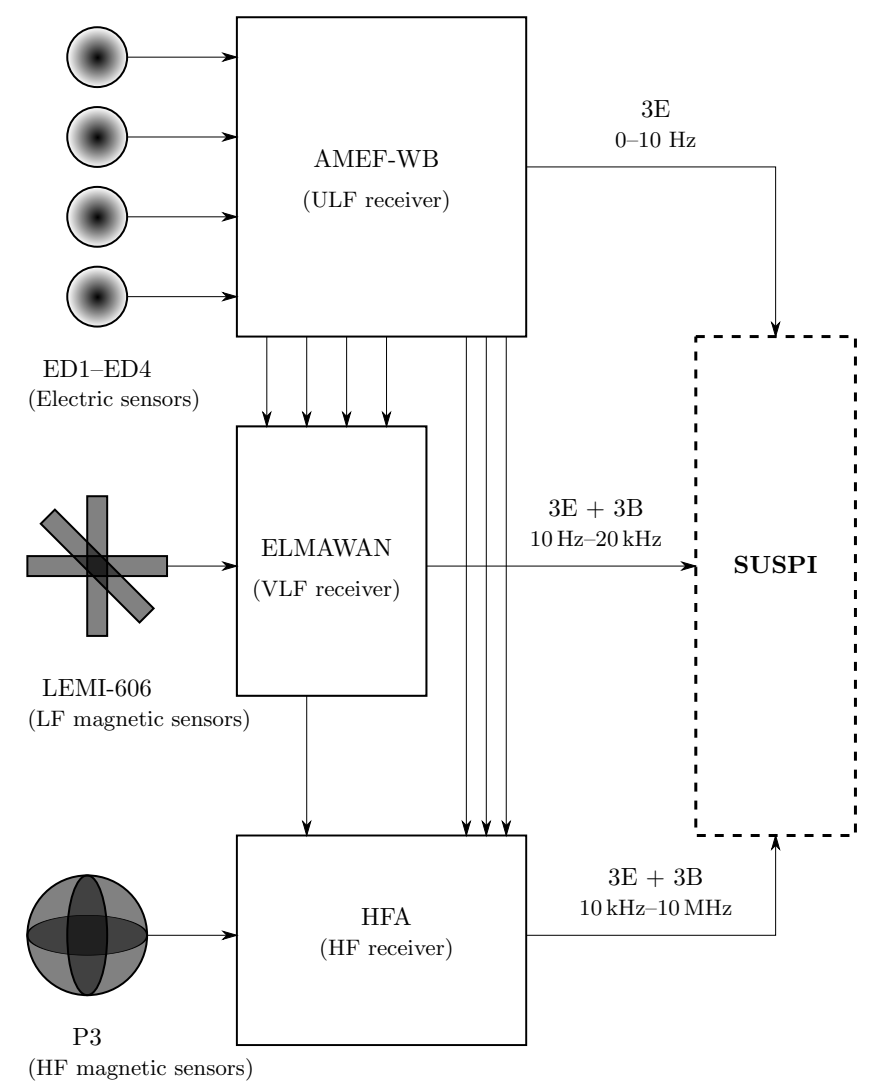

Figure 1. Block diagram of the RESONANCE particle and plasma wave complex.

tion (Vogl et al., 2004; Cecconi and Zarka, 2005) are practically unfeasible. In the evaluation of spaceborne radio astronomy observations, the preferred quantity to describe the antenna is the effective length vector $\boldsymbol{h}_{\mathrm{e}}$. However, around and above the first antenna resonance, this vector is intricate and cumbersome to use, because it becomes a complexvalued, direction-dependent quantity above the quasi-static frequency range (above some $\mathrm{MHz}$ ).

The presented calculations are based on solving the underlying integral equations using the well-known numerical programs ASAP (McCormack, 1974) and CONCEPT-II (TU Hamburg-Harburg, 2010). In the course of the project a larger number of configuration options were investigated, from simple wire-grid models up to full patch models, as well as different options for satellite configuration details. The applied spherical spacecraft coordinate system definition is shown in Fig. 2. We already outlined in Sampl et al. (2012) that wiregrid representations of the simulation model are inappropriate, since they result in significant inaccuracies, especially for the small cylindrical sensors. So the final results shown in this contribution were calculated with CONCEPT-II and our numerical reference model (Figs. 3, 4), which is fully composed of surface patches. All other analyzed configurations can be found in Macher et al. (2009). CONCEPT-II solves the 


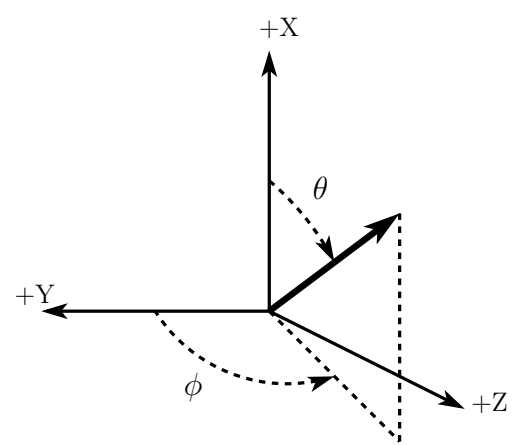

Figure 2. Definition of spherical coordinates $\theta$ (colatitude) and $\phi$ (azimuth) in the spacecraft-fixed reference frame as used for the representation of antenna axes.

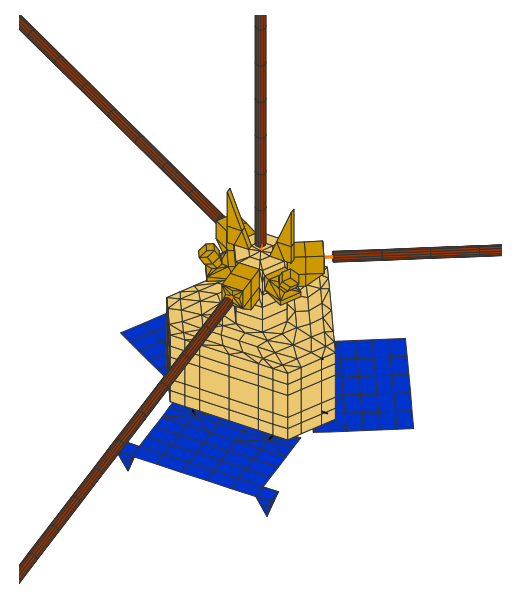

Figure 3. Final, detailed patch-grid model of the RESONANCE spacecraft, oblique view.

underlying electric field integral equation (EFIE) (Balanis, 2005) by applying the "method of moments" (MoM) (Mader, 1992). Using this method the EFIE is rewritten by expansion with a finite number of weighted basis functions into a system of linear equations, which can then can be solved by linear algebra. More detailed solution approaches can be found in Harrington (1968), Wang (1990), and Schroth (1985) and respective literature.

\section{Quantities for the characterization of the system}

Above the quasi-static range the effective length vector changes with wave incident direction and frequency. Furthermore, the sensor system is generally not purely capacitive anymore, so the impedance matrix cannot be represented in the form $\mathbf{Z}=(j \omega \mathbf{C})^{-1}$ in terms of a real capacitance matrix $\mathbf{C}$. We therefore have to consider other parameters to quantify and illustrate the reception properties. For that purpose we use the antenna effective area and the elements of the impedance matrix $\mathbf{Z}$. The former is always a real value,
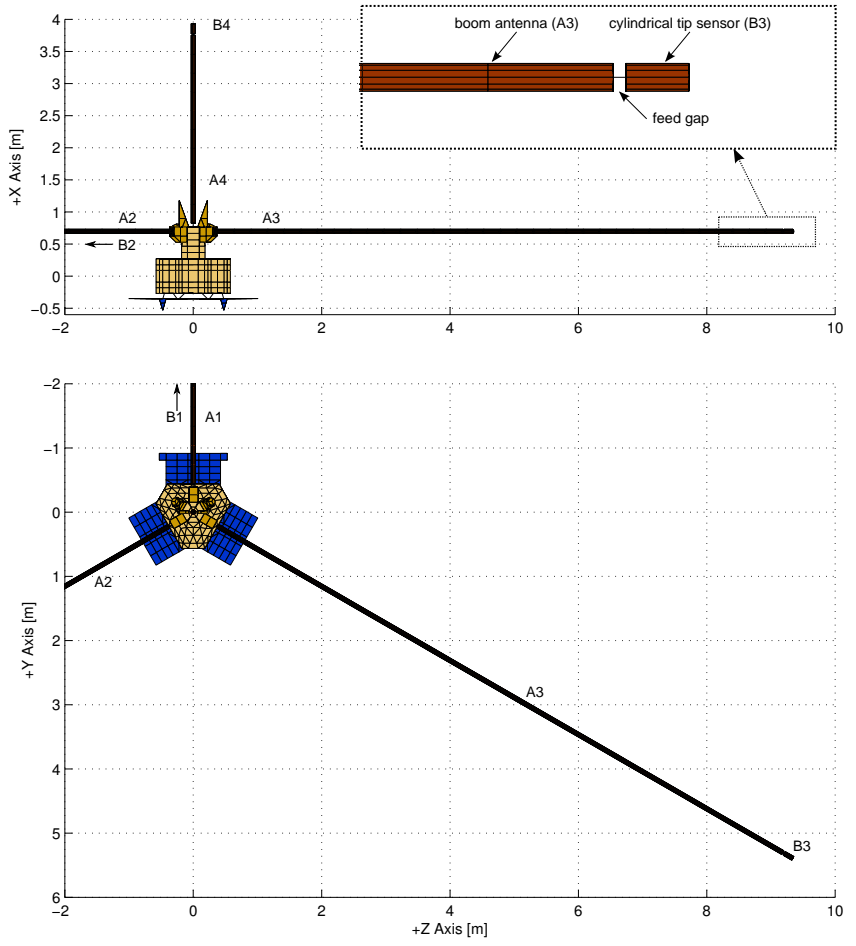

Figure 4. Final, detailed patch-grid model of the RESONANCE spacecraft, top and front view.

whereas the latter is generally complex (with a purely imaginary limit for $\omega \rightarrow 0$ ). We define the effective area $A$ of an antenna as

$A=\frac{|V|^{2}}{|\mathbf{E}|^{2}}$

where $V$ is the received voltage and $\mathbf{E}$ the electric field strength of the incident plane wave. Polarization matching is assumed, i.e., the conjugate of $\mathbf{E}$ is proportional to the effective length vector $\boldsymbol{h}_{\mathrm{e}}$, as defined by Macher et al. (2009). This definition of $A$ is slightly different from the usual text book definition (Balanis, 2005) and adapted to the measurement techniques and data evaluation methods applied in the present context, which rely on open-port voltages instead of power values. The usual definition refers to the received power per incident power flux (assuming polarization and impedance matching), which is of no use in the present context, it is even invalid for open ports.

Figures 5 and 6 are dedicated to effective area patterns $A$ for frequencies around the antenna resonances. The color scale is logarithmic, with $0 \mathrm{~dB}$ at $500 \mathrm{~m}^{2}$ for the upper three patterns $(A 1-A 3, B 1-B 3)$ and $0 \mathrm{~dB}$ at $50 \mathrm{~m}^{2}$ for the lowest pattern ( $A 4$ and $B 4)$. With this normalization the maximum effective area in the frequency range 0 to $+40 \mathrm{MHz}$ maps approximately to the same color index (dark red) for all sensor types. This is plausible since the length of the fourth boom (A4) is approximately a third of the long booms 

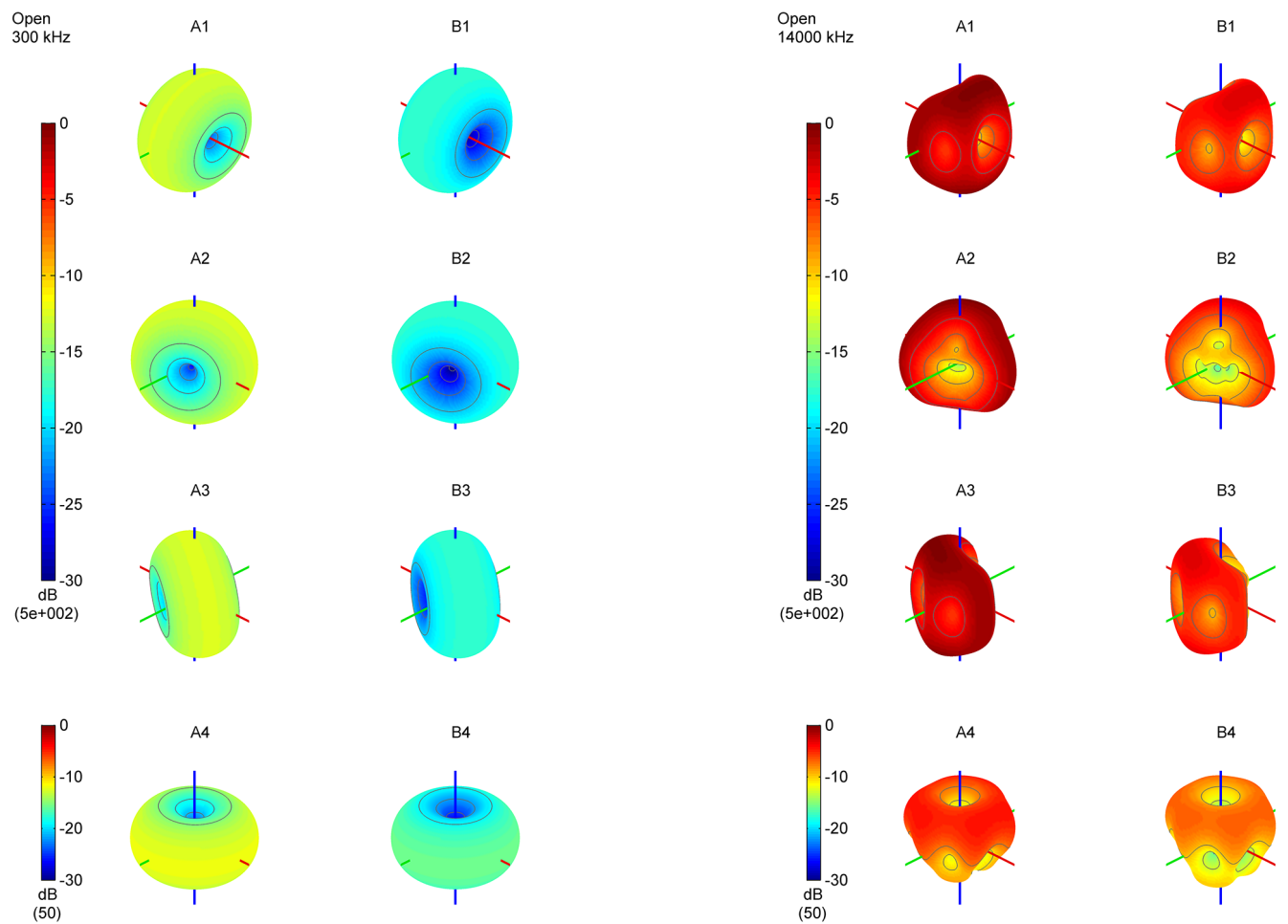

Figure 5. Effective area patterns of boom antennas $A 1-A 4$ and short cylindrical sensors $B 1-B 4$ at $300 \mathrm{kHz}$ (left) and $14 \mathrm{MHz}$ (right). The panels show an oblique view, with the $+x$ axis pointing upwards, the $+y$ axis to the right and towards the observer, and the $+z$ axis to the right and away from the observer The color scale is at $0 \mathrm{~dB} / 500 \mathrm{~m}^{2}$ for $A 1-A 3$ and $B 1-B 3$ and at $0 \mathrm{~dB} / 50 \mathrm{~m}^{2} A 4$ and $B 4$.
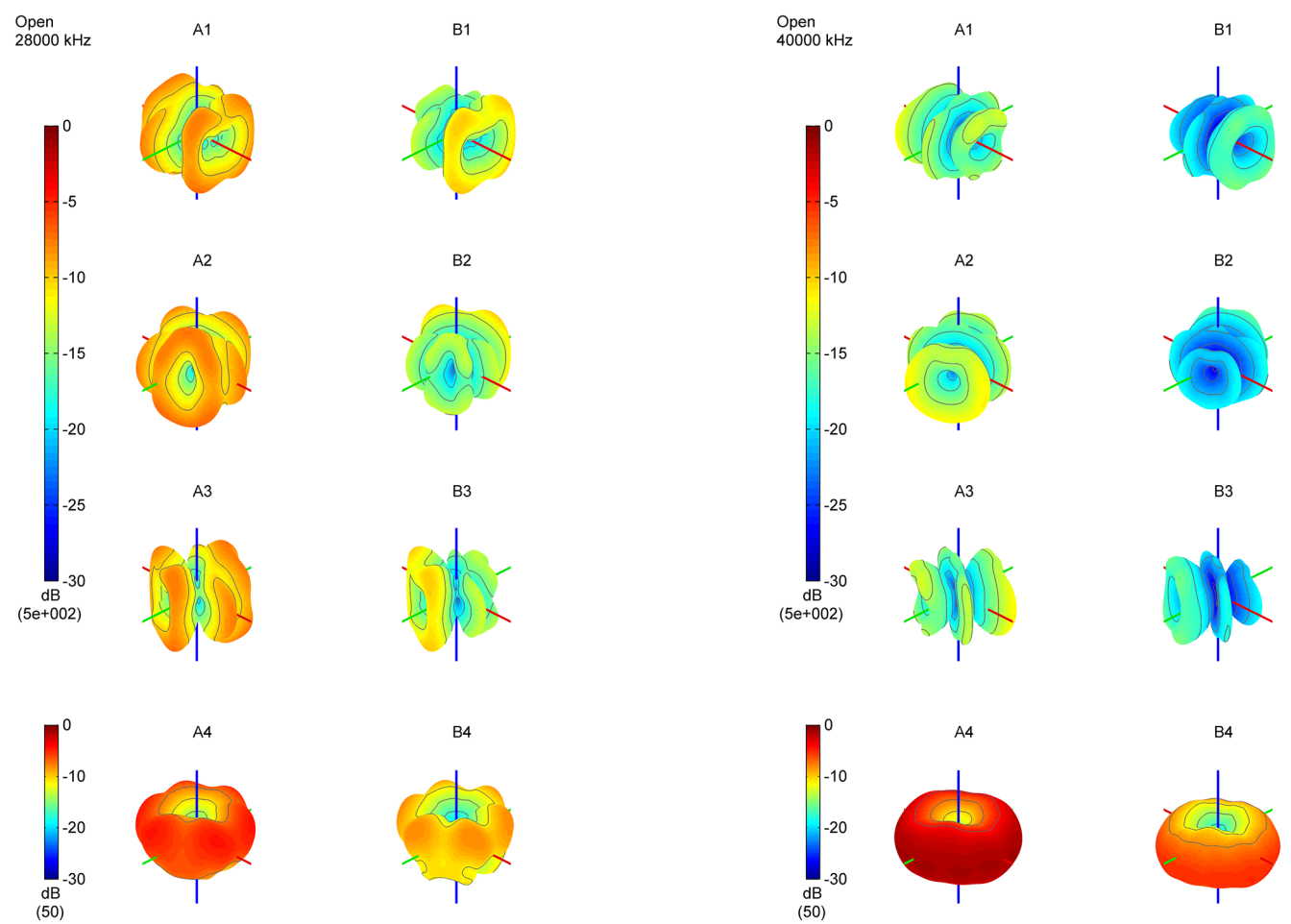

Figure 6. Effective area patterns analogous to Fig. 5, but for $28 \mathrm{MHz}$ (left) and $40 \mathrm{MHz}$ (right). 
(A1-A3), so the ratio of the respective effective areas is about a tenth, which explains the difference in the dB-reference values $\left(50 \mathrm{~m}^{2}\right.$ vs. $\left.500 \mathrm{~m}^{2}\right)$.

The first illustrated frequency is $300 \mathrm{kHz}$ (Fig. 5, left panel), which is representative of the quasi-static range. We can clearly recognize the torus-like form of the patterns. The symmetry axes of the tori coincide with the directions of the respective quasi-static effective length vectors as shown in our related paper, (Sampl et al., 2012). The maximum effective areas of the $B$-antennas are smaller than those of the $A$-antennas. We can verify this for the $n$th antenna boom by calculating the ratio of the squares of the effective length vectors $\boldsymbol{h}_{B_{n}}$ and $\boldsymbol{h}_{A_{n}}$ by

$$
\frac{\max \left(A_{B_{n}}\right)}{\max \left(A_{A_{n}}\right)}=\frac{\left|\boldsymbol{h}_{B_{n}}\right|^{2}}{\left|\boldsymbol{h}_{A_{n}}\right|^{2}} \text {. }
$$

This formula can be derived from the fact that the effective area is connected with the effective length vector via $A=$ $|\boldsymbol{e} \times \boldsymbol{h}|^{2}$, with $\boldsymbol{e}$ being the unit vector pointing in the direction where the incident plane wave comes from. For instance, with $n=1$ we get the ratio $\max \left(A_{B_{1}}\right) / \max \left(A_{A_{1}}\right)=0.31$ in the quasi-static range, using values from Table I in Sampl et al. (2012), which means a difference of about $-5 \mathrm{~dB}$ between $A$ - and $B$-antennas appearing in the color scale of the patterns.

\section{Sensor impedances}

Figures 7 and 8 contain impedance curves, exhibiting the dependence of the elements of the impedance matrix $\mathbf{Z}$ on frequency.

Figure 7 presents the (self-) impedances, i.e., the diagonal elements of the respective matrix, of the $A$ - and $B$-antennas $\left(A_{n}-A_{n}\right.$ and $B_{n}-B_{n}$ for $\left.n=1 \ldots 4\right)$. Figure 8, left panel, is dedicated to the mutual impedances of $A$-antennas with other $A$-antennas $\left(A_{n}-A_{m}\right.$ for $n, m=1 \ldots 4$ with $\left.n \neq m\right)$. Figure 8, right panel, shows the corresponding curves for $B$ antennas $\left(B_{n}-B_{m}\right.$ for $\left.n, m=1 \ldots 4 ; n \neq m\right)$. All other combinations of mutual impedances/admittances of $A$-antennas with $B$-antennas on the same boom $\left(A_{n}-B_{n}\right)$ and on different booms $\left(A_{n}-B_{m} ; n, m=1 \ldots 4 ; n \neq m\right)$ are not shown, but can be found in Macher et al. (2009).

To show the antenna systems mutual impedances only one half of the off-diagonal elements of impedance matrix $\mathbf{Z}$ need to be depicted. $\mathbf{Z}$ is symmetrical due to the antenna systems reciprocity (Macher, 2012, 2014) and the other half gives the same curves again - apart from numerical inaccuracies. Many curves overlap due to the symmetry of the satellite geometry; they are plotted in the same color.

In the impedance plots we recognize resonances at 14 and $28 \mathrm{MHz}$ (q.v. Figs. 5 and 6), best visible as maxima in the real part in Fig. 7. They are very pronounced for the antennas $A 1-A 3$ and $B 1-B 3$, but very faint if only $A 4$ and $B 4$ are involved $\left(\mathbf{Z}_{A 4, A 4}, \mathbf{Z}_{B 4, B 4}\right.$, and $\left.\mathbf{Z}_{A 4, B 4}\right)$. These two resonances appear at the frequencies where the boom length agrees with the half-wavelength $(\lambda / 2$-resonance) and the full wavelength ( $\lambda$-resonance). Therefore, the $\lambda / 2$-resonance associated with the short boom (antennas $A 4 / B 4$ ) can be expected at about $\frac{300}{2 \cdot 3.3} \approx 45 \mathrm{MHz}$, where $3.3 \mathrm{~m}$ is the full boom length including mounting. It is approached at the end of the exhibited frequency interval. The $\lambda / 4$-resonance associated with the long booms is to be expected at about $\frac{300}{4 \cdot 10.3} \approx 7 \mathrm{MHz}$, and that of the short boom at $\frac{300}{4 \cdot 3.3} \approx 22 \mathrm{MHz}$. The displayed frequency interval contains only the first resonance $(\lambda / 4)$ for the short boom, but it contains all the resonances up to the fifth (at $\approx 35 \mathrm{MHz}$ ) for the long booms. The third and fourth resonance $(\approx 21 \mathrm{MHz}$ and $\approx 28 \mathrm{MHz})$ of antennas associated with the long booms are more pronounced in the admittance plots. They are identifiable as zeros of $\Im(\mathbf{Z})$ in Fig. 7. The reason for the deviation of the patterns from the respective ideal dipole characteristic is the radiation coupling with the satellite body and also between the antennas. With the same reasoning we can see why it is plausible that the $\lambda / 2$ resonance of the long booms is split in two impedance maxima.

In the mutual impedance (q.v. Fig. 8) and mutual admittance curves the resonances of both correlated antennas have their effect, so these curves are generally rather intricate. Even more since the real parts of the mutual impedances may be negative; actually they typically change signs close to the $\lambda / 2$ and $\lambda$-resonances (except for $\mathbf{Z}_{A i B i} ; i=1 \ldots 4$ ). In contrast, the real parts of the self-impedances are always positive as they are representative of the power input to the antennas when operated in particular transmission modes.

\section{Effective area pattern}

Having identified the resonance frequencies, we can utilize this knowledge for a better understanding of the effective area patterns as shown in Figs. 5 and 6.

In the plots the principal axes are drawn in red ( $y$ axis parallel to $A 1$ and $B 1$ ), green ( $z$ axis) and blue ( $x$ axis parallel to $A 4$ and $B 4$ ). The panels show an oblique view, with the $+x$ axis pointing upwards, the $+y$ axis to the right and towards the observer, and the $+z$ axis to the right and away from the observer.

When we increase the frequency above the quasi-static range the toroidal shapes of the antenna patterns change. However, there is nearly no change of the shape up to $5 \mathrm{MHz}$, only the magnitudes increase. At $10 \mathrm{MHz}$ the patterns get dented, but are still of toroidal shape. First we consider the antennas $A 1 / B 1-A 3 / B 3$. The closer the frequency gets to the $\lambda / 2$-resonance at $14 \mathrm{MHz}$ (Fig. 5, right) the more their pattern is changed, finally taking a completely different form at the resonance frequency. Above the $\lambda / 2$-resonance a pattern composed of two toroids develops (similar to an aircraft wheel), which remains up to the $\lambda$-resonance, where again a complete pattern change occurs. 

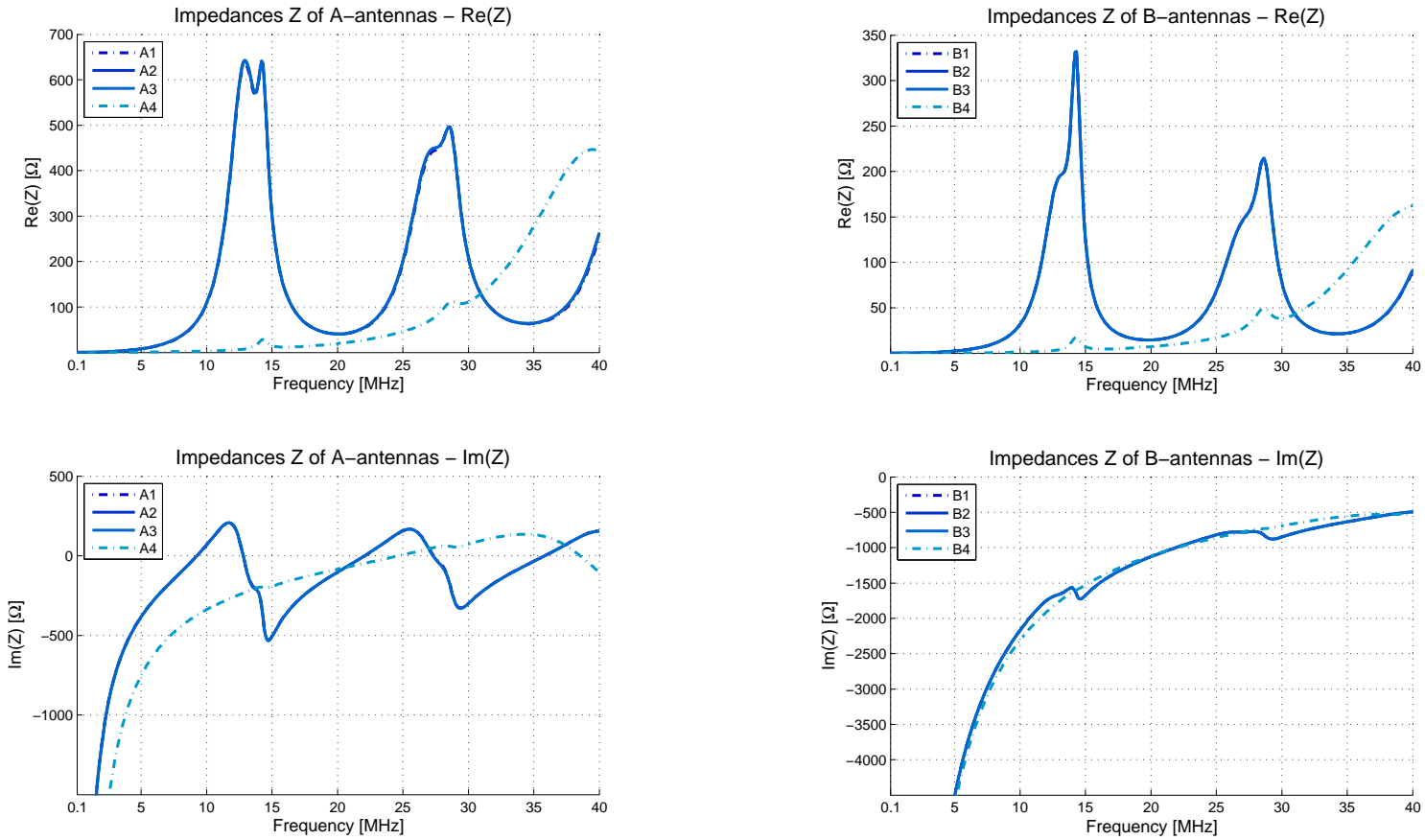

Figure 7. Self-impedances of the boom antennas $A 1-A 4$ (left) and the short cylindrical sensors $B 1-B 4$ (right). The shown quantities are the diagonal elements of the impedance matrix $\mathbf{Z}$ as a function of frequency. Upper panels contain the real parts, lower panels the imaginary parts. The curves for the boom antennas $A 1-A 3$ and sensor $B 1-B 3$ are nearly identical, which is due to the high symmetry of their deployment on the satellite.
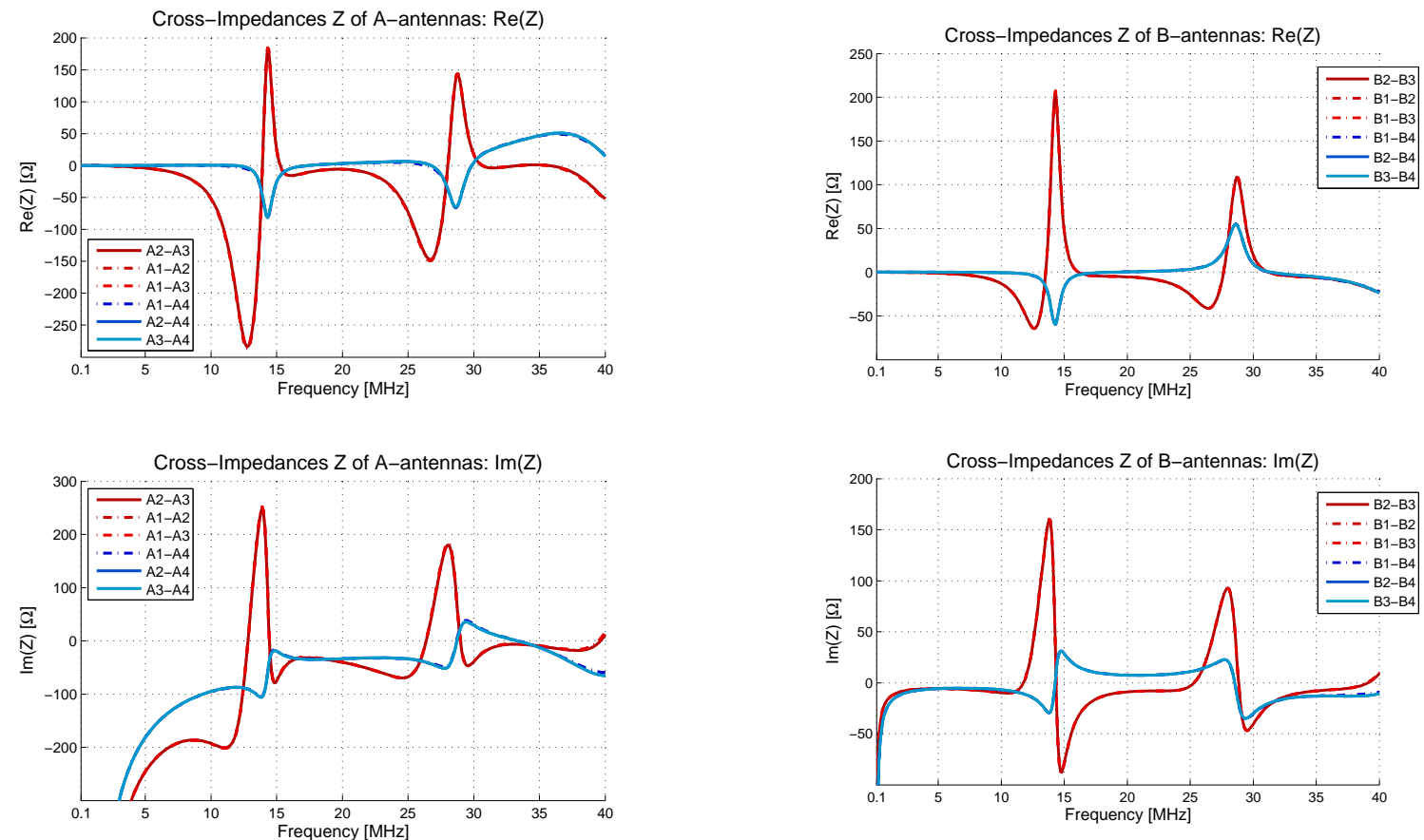

Figure 8. Mutual impedances of the boom antennas $A 1-A 4$ (left) and the short cylindrical sensors $B 1-B 4$ (right). The shown quantities are the off-diagonal elements of the impedance matrix $\mathbf{Z}$, as a function of frequency. Upper panels contain the real parts, lower panels the imaginary parts. The red curves overlap due to the symmetry of the mounting of the booms $A 1-A 3$ and $B 1-B 3$. The same holds for the blue curves since $A 4$ and $B 4$ are in the symmetry axis of the satellite. 
Above the $\lambda$-resonance a transition region follows where the pattern transforms into a shape composed of three toroidal lobes.

At the $\lambda / 2$ and $\lambda$-resonance the antennas perform best in the sense that they provide the highest gain in transmission mode and the highest sensitivity (with largest effective area) when receiving. But the quite irregular patterns at these resonances do not admit an accurate prediction of the reception dependence on the direction of incidence, so direction finding is practically impossible at these frequencies. Off the resonance frequencies gain and effective area are smaller but we can reckon with a rather regular pattern (as mentioned above a simple toroid or a shape composed of two or three toroids in the frequency range of interest). The mentioned $\lambda / 2$ and $\lambda$-resonances are also visible in the effective area patterns of $A 4$ and $B 4$, causing a distortion of the toroidal form. The own $\lambda / 2$-resonance of $A 4 / B 4$ antennas $(\approx 45 \mathrm{MHz})$ distorts their pattern at the end of the investigated frequency interval $(40 \mathrm{MHz}$, Fig. 6, right), but is too far away to deform the toroid completely.

All investigations in this project are done for open ports, so it is assumed that the preamplifiers or receivers connected to the antennas have very high input impedances. If these impedances are not sufficiently high (of the order of $10 \mathrm{M} \Omega$ or higher) or cable capacitances are significant, one cannot speak of open-port operation anymore. In such a case the base impedances have to be taken into account. This can change the results significantly, in particular near the resonance frequencies, as Gurnett et al. (2004), Macher et al. (2007) and Bale et al. (2008) have already shown in the context of former spaceborne antennas.

\section{Conclusions}

In this contribution we outline the properties above the quasi-static range of the space-borne electric field sensors as planned for the science mission "RESONANCE". The reception patterns, self- and mutual impedances of boom antennas $(A 1-A 4)$ and short cylindrical sensors $(B 1-B 4)$ are calculated from the results of numerical computations, covering the whole instrument's frequency range (from near DC to $40 \mathrm{MHz}$ ) with $100 \mathrm{kHz}$ step size. Provided effective area patterns are of the typical toroidal shape in the quasi-static frequency range. The toroids get more and more distorted when increasing the frequency and adopt completely different, quite peculiar, shapes around the resonances. The provided reception patterns give a visual estimate of the overall reception properties, in particular how the effective areas, and the receiving sensitivity, of the antennas depend on the frequency and the direction of wave incidence.

Acknowledgements. The authors want to thank Mikhail Yanovsky of the Russian Space Research Institute for the invaluable information about the RESONANCE spacecraft design, and Jean-
Louis Rauch from the Laboratoire de Physique et Chimie de l'Environnement (CNRS) for information on the cylindrical tip sensors.

This work is part of the science project "RESONANCE electric field sensors: determination of the optimum configuration", which was financed by the Austrian Research Promotion Agency (FFG) in the framework of ASAP 4, project 816159.

Edited by: V. Korepanov

\section{References}

Balanis, C. A.: Antenna Theory: Analysis and Design, John Wiley \& Sons, Inc., Hoboken, New Jersey, USA, 3rd Edn., 2005.

Bale, S., Ullrich, R., Goetz, K., Alster, N., Cecconi, B., Dekkali, M. Lingner, N., Macher, W., Manning, R., McCauley, J., Monson, S., Oswald, T., and Pulupa, M.: The Electric Antennas for the STEREO/WAVES Experiment, Space Sci. Rev., 136, 529-547, doi:10.1007/s11214-007-9251-x, 2008.

Boudjada, M., Galopeau, P., Mogilevski, M., Lecacheux, A., Kuril'chik, V. N., and Rucker, H.: RESONANCE Project: Comparative studies of observational features associated to auroral radio emissions, in: The Inner Magnetosphere and the Auroral Zone PhysicsThe Inner Magnetosphere and the Auroral Zone Physics, Space Research Institute, Moscow, Russia, Moscow, 2010.

Cecconi, B. and Zarka, P.: Direction finding and antenna calibration through analytical inversion of radio measurements performed using a system of two or three electric dipole antennas on a three-axis stabilized spacecraft, Radio Sci., 40, RS3003, doi:10.1029/2004RS003070, 2005.

Demekhov, A. G., Trakhtengerts, V. Y., Mogilevsky, M. M., and Zelenyi, L. M.: Current problems in studies of magnetospheric cyclotron masers and new space project "Resonance", Adv. Space Res., 32, 355-374, doi:10.1016/S0273-1177(03)90274-2, 2003.

Gurnett, D. A.: Principles of Space Plasma Wave Instrument Design, in: Measurement Techniques in Space Plasmas - Fields, edited by: Pfaff, R. F., Borovsky, J. E., and Young, D. T., Geophysical Monograph, American Geophysical Union, Washington, D.C., 1998.

Gurnett, D. A., Kurth, W. S., Kirchner, D. L., Hospodarsky, G. B., Averkamp, T. F., Zarka, P., Lecacheux, A., Manning, R., Roux, A., Canu, P., Cornilleau-Wehrlin, N., Galopeau, P., Meyer, A., Boström, R., Gustafsson, G., Wahlund, J.-E., Åhlen, L., Rucker, H. O., Ladreiter, H. P., Macher, W., Woolliscroft, L. J. C., Alleyne, H., Kaiser, M. L., Desch, M. D., Farrell, W. M., Harvey, C. C., Louarn, P., Kellogg, P. J., Goetz, K., and Pedersen, A.: The Cassini Radio and Plasma Wave Investigation, Space Sci. Rev., 114, 395-463, doi:10.1007/s11214-004-1434-0, 2004.

Harrington, R. F.: Field computation by moment methods, Malabar, FL: Krieger, 1968.

Macher, W.: Inter-Reciprocity Principles for Linear NetworkWaveguides Systems Based on Generalized Scattering, Admittance and Impedance Matrices, Circuits and Systems I: Regular Papers, IEEE Trans., 59, 721-734, doi:10.1109/TCSI.2011.2169888, 2012. 
Macher, W.: Transfer operator theory and inter-reciprocity of nonreciprocal multiport antennas, Progr. Electromag. Res. B, 60, 169-193, doi:10.2528/PIERB14051401, 2014.

Macher, W., Oswald, T., Fischer, G., and Rucker, H. O.: Rheometry of multi-port spaceborne antennas including mutual antenna capacitances and application to STEREO/WAVES, Measure. Sci. Technol., 18, 3731-3742, 2007.

Macher, W., Sampl, M., Gruber, C., Oswald, T., and Rucker, H. O.: Resonance electric field sensors. Report on the ASAP4-Project, Technical Report IWF-183, Space Research Institute, Austrian Academy of Sciences, Austrian Academy of Sciences, Graz, Austria, 2009.

Mader, T.: Berechnung elektromagnetischer Felderscheinungen in abschnittsweise homogenen Medien mit Oberflächenstromsimulation, PhD thesis, Technische Universität Hamburg-Harburg, 1992.

McCormack, J.: Antenna Analysis: Antenna Scatterers Analysis Program, available at: http://raylcross.net/asap/ (last access: 8 September 2008), 1974.

Mogilevsky, M. M., Zelenyi, L., Trakhtengerts, V., Demekhov, A., Sukhanov, K., Sheikhet, A., Yanovsky, M., Romantsova, T., Rauch, J.-L., Parrot, M., F.Lefeuvre, Bosinger, T., Rietveld, M., Galeev, A., Burinskaya, T., Vaisberg, O., Smirnov, V., Nazarov, V., Kudryshov, V., Lichachev, V., Rusanov, A., Morozova, E., Savin, S., Sadovsky, A., Aleksandrova, T., Pasmanik, D., Baum, F., Kaurova, I., Batanov, O., Melnik, A., and Kharchenko, G.: Resonance Project. Study of wave-particle interaction and plasma dynamics in the inner magnetosphere. REPORT ON THE PHASE A., Tech. Rep. 54-R/26-2-131/R-E, Space Research Institute, 84/32 Profsoyuznaya Str., 117997, Moscow, Russia, 2002.
Mogilevsky, M. M., Zelenyi, L. M., Demekhov, A. G., Petrukovich, A. A., and Shklyar, D. R.: RESONANCE Project for Studies of Wave-Particle Interactions in the Inner Magnetosphere, Am. Geophys. Union, 199, 117-126, doi:10.1029/2012GM001334, 2013.

Oswald, T., Macher, W., Rucker, H., Fischer, G., Taubenschuss, U., Bougeret, J., Lecacheux, A., Kaiser, M., and Goetz, K.: Various methods of calibration of the STEREO/WAVES antennas, Adv. Space Res., 43, 355-364, 2009.

Rucker, H. O., Macher, W., Manning, R., and Ladreiter, H. P.: Cassini model rheometry, Radio Sci., 31, 1299-1311, 1996.

Sampl, M., Macher, W., Gruber, C., Oswald, T., Rucker, H., and Mogilevsky, M.: Calibration of Electric Field Sensors Onboard the Resonance Satellite, Antennas and Propagation, IEEE Trans., 60, 267-273, doi:10.1109/TAP.2011.2167918, 2012.

Schroth, A.: Moderne numerische Verfahren zur Lösung von Antennen- und Streuproblemen, Oldenbourg, 1985.

TU Hamburg-Harburg: CONCEPT-II, available at: http://www.tet. tu-harburg.de/concept, last access: 12 October 2010.

Vogl, D. F., Cecconi, B., Macher, W., Zarka, P., Ladreiter, H. P., Fedou, P., Lecacheux, A., Averkamp, T., Fischer, G., Rucker, H. O., Gurnett, D. A., Kurth, W. S., and Hospodarsky, G. B.: In-flight calibration of the Cassini-Radio and Plasma Wave Science (RPWS) antenna system for direction-finding and polarization measurements, J. Geophys. Res. Space Phys., 109, A09S17, doi:10.1029/2003JA010261, 2004.

Wang, J. J. H.: Generalised moment methods in electromagnetics, IEE Proceedings H Microwaves, Antenn. Propa., 137, 127-132, 1990. 